\title{
MIMO Interference Management Using Precoding Design
}

\author{
Martin Crew ${ }^{1}$, Osama Gamal Hassan ${ }^{2}$ and Mohammed Juned Ahmed ${ }^{3}$ \\ ${ }^{1}$ University of Cape Town, South Africa \\ martincrewetopmail.co.za \\ ${ }^{2}$ Cairo University, Egypt \\ ${ }^{3}$ King Abdullah University of Science and Technology, Saudi Arabia
}

\begin{abstract}
In this paper, we investigate how to design precoders to achieve full diversity and low decoding complexity for MIMO systems. First, we assume that we have 2 transmitters each with multiple antennas and 2 receivers each with multiple antennas. Each transmitter sends codewords to respective receiver at the same time. It is difficult to handle this problem because of interference. Therefore, we propose an orthogonal transmission scheme that combines space-time codes and array processing to achieve lowcomplexity decoding and full diversity for transmitted signals. Simulation results validate our theoretical analysis.
\end{abstract}

\section{KEYWORDS}

Z Channel, Alamouti Codes, MIMO, Interference Cancellation, Complexity, Co-channel Interference.

\section{INTRODUCTION}

Multiple-input multiple-output (MIMO) channels arise in many different scenarios such as when a bundle of twisted pairs in digital subscriber lines (DSLs) is treated as a whole, when multiple antennas are used at both sides of a wireless link, or simply when a frequencyselective channel is properly modeled by using, for example, transmit and receive filterbanks. In particular, MIMO channels arising from the use of multiple antennas at both the transmitter and at the receiver have recently attracted significant interest because they provide an important increase in capacity over single-input single-output (SISO) channels under some uncorrelation conditions [1-6].

Recently, several space-time processing techniques have been used in multiple access channels to reduce the decoding complexity and enhance system performance by cancelling the interference from different users [7-10]. When it comes to Z channels [11], a scenario when there are two users each transmitting different codewords to two receivers simultaneously, how to achieve low-complexity decoding and high performance such as full diversity is still an open problem.

In this paper, we investigate how to achieve the low-complexity decoding and the highest possible diversity to improve the transmission quality for space-time codes in Zrate feedback channel. This is not unreasonable; control channels are often available to implement power control, adaptive modulation, and certain closed-loop diversity modes. 


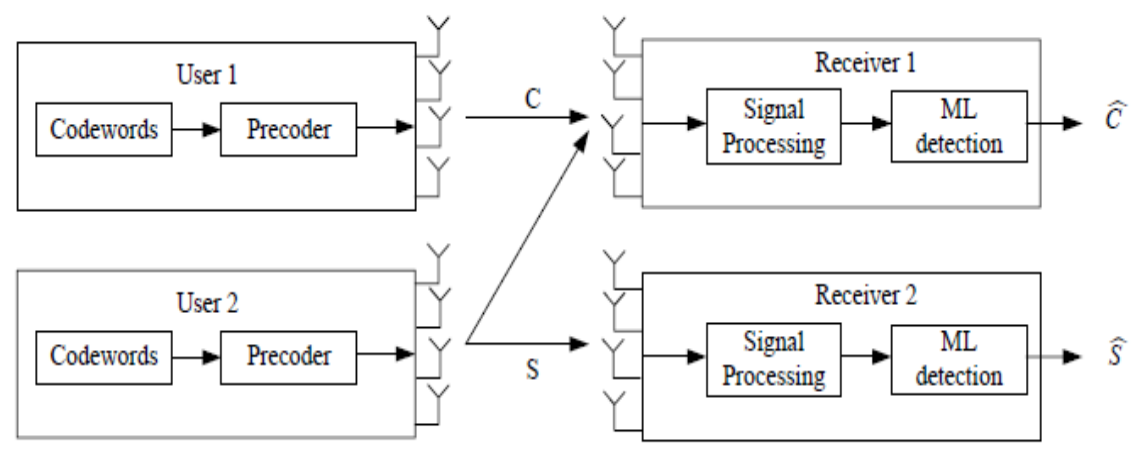

Figure 1: Z Channel

channels without losing symbol rate. We assume that our system operates under short term power constraints, fixed codeword block length and limited delay. Under these constraints, there will always be some outage probability [12-15]. For example, [14] shows that outage probability exisits for the block-fading channel with limited delay and block length. [15] points out that when the delay is finite, for any finite rate, as small as it may be, there is a nonzero outage probability independent of the code length. Thus, the diversity is an important tool to evaluate the system performance.

The outline of the paper follows next. Section II introduces our motivation and the $\mathrm{Z}$ channels we discuss in this paper. In Section III, we propose an orthogonal transmission scheme which is necessary to achieve low complexity decoding, high coding gain and full diversity as shown in later sections. In Section IV, our decoding scheme is proposed. We analyze the performance of our scheme in Section V. Simulation results are presented in Section VI and Section VII concludes the paper.

Notation: We use boldface letters to denote matrices and vectors, super-scripts $(\cdot) T,(\cdot) *,(\cdot) \dagger$ to denote transpose, conjugate and transpose conjugate, respectively. We denote the element in the $i$ th row and the $j$ th column of matrix $\mathbf{X}$ by $X(i, j)$. We denote the $j$ th column of a matrix $\mathbf{X}$ by $\mathbf{X}(j)$.

\section{Motivation and Channel Model}

We assume there are 2 users each with 4 transmit antennas and 2 receivers each with 4 receive antennas. Both users want to send different space-time codes to Receivers 1 and 2 on the same frequency band at the same time. As shown in Figure 1, User 1 wants to send codeword $C$ to Receiver 1 without causing interference to Receiver 2. User 2 wants to send codeword $S$ to Receiver 2 and causes interference to Receiver 1. When channel knowledge is not available at the transmitters, space-time codes combined with TDMA can be used to achieve symbol-bysymbol decoding and full diversity. But the symbol rate reduces to one half. A solution to keep the symbol rate unchanged when space-time codes are used, is to combine space-time coding and array processing. In other words, we allow all transmitters to send space-time codes simultaneously to keep rate one and utilize special array processing techniques to achieve lowcomplexity decoding and full diversity. In this paper, we achieve the above goals under shortterm power constraints, fixed codeword block length and limited delay, when there is outage. We do not claim that our scheme can achieve capacity or full degree of freedom. After all, there is a tradeoff between diversity and multiplexing gain, which is outside scope of this paper. We introduce input-output equations. We let each user transmit Alamouti Codes [17] as follows:

$$
\mathbf{C}=\left(\begin{array}{cc}
c_{1} & -c_{2}^{*} \\
c_{2} & c_{1}^{*}
\end{array}\right), \quad \mathbf{S}=\left(\begin{array}{cc}
s_{1} & -s_{2}^{*} \\
s_{2} & s_{1}^{*}
\end{array}\right)
$$


where $i, j=1,2$. Note that we can also use other space-time codes with rate one and Alamouti code is just one example. Let

$$
\mathbf{A}^{\mathrm{t}}=\mathbf{A}^{\mathrm{t}}(4,4)
$$

be the precoders we need to design. In this paper, we use $(i, j)$ denote a matrix of $i \times j$ dimension. They are combined with the space-time codes sent by User 1 and this is the first step of our array processing technique. Note that in order to satisfy the short-term power constraint, we need

$$
\left\|\mathbf{A}^{t}(4,4)\right\|_{F}^{2}=1
$$

Similarly, the precoders for User 2 is defined as

$$
\mathbf{B}^{\mathrm{t}}=\mathbf{B}^{\mathrm{t}}(4,4)
$$

with the power constraint

$$
\left\|\mathbf{B}^{t}(4,4)\right\|_{F}^{2}=1
$$

The channels are quasi-static flat Rayleigh fading and keep unchanged during two time slots. Then we let

$$
\mathbf{H}_{l}=\mathbf{H}_{l}(4,4)
$$

denote the channel matrix between User 1 and Receivers $l$, respectively. Similarly, we use

$$
\mathbf{G}_{l}=\mathbf{G}_{l}(4,4)
$$

to denote the channel matrix between User 2 and Receiver $l$, respectively. Then the received signals at Receiver 1 at time slot $t$ can be denoted by

$$
\mathbf{y}_{1}^{t}(4,1)=\mathbf{H}_{1}(4,4) \mathbf{A}^{t}(4,4) \mathbf{C}(t)+\mathbf{G}_{1}(4,4) \mathbf{B}^{t}(4,4) \mathbf{S}(t)+\mathbf{n}_{1}^{t}(4,1)
$$

Where

$$
\mathbf{y}_{1}^{t}=\mathbf{y}_{1}^{t}(4,1), \quad \mathbf{n}_{1}^{t}=\mathbf{n}_{1}^{t}(4,1)
$$

denote the received signals and the noise at Receiver 1, respectively, at time slot $t$. Similarly, at time slot $t$, Receiver 2 will receive the following signals

$$
\mathbf{y}_{2}^{t}(4,1)=\mathbf{G}_{2}(4,4) \mathbf{B}^{t}(4,4) \mathbf{S}(t)+\mathbf{n}_{2}^{t}(4,1)
$$

Where

$$
\mathrm{y}_{2}^{t}=\mathrm{y}_{2}^{t}(4,1), \quad \mathrm{n}_{2}^{t}=\mathrm{n}_{2}^{t}(4,1)
$$

Equations (8) and (10) are the channel equations on which we will base our design in this paper.

\section{Precoder Design and Orthogonal Transmission Structure}

In this section, we will build an orthogonal transmission structure by combining the space-time codes and our precoders. This orthogonal transmission structure is necessary because it provides two benefits. The first benefit is that low-complexity decoding can be realized because under this orthogonal transmission structure, different codewords will be sent along different orthogonal vectors. We can easily decode the symbols without the interference at each receiver. The second benefit is that we can achieve full diversity and higher coding gain once we make 
the proper array processing as shown in later sections. This is the key difference between our array processing method and the interference alignment method. The latter can only achieve the first benefit. Of course, the tradeoff is that we lose the maximum possible degree of freedom in the process.

Different users and different codewords may have different diversities. By saying full diversity, we mean the diversity is full for each codeword sent by each user. For example, full diversity for User 1 means at Receiver 1, the diversity for codeword $\mathbf{C}$ is full. Similarly, by saying the diversity for User 2 is full, we mean that at Receiver 2, the diversity for codeword $\mathbf{S}$ is full. In this section, we show how to build the orthogonal transmission structure by designing proper precoders. Later, we will show that our proposed orthogonal transmission scheme can achieve low-complexity decoding and full diversity.

Our main idea to build the orthogonal transmission structure is to adjust each signal in the signal space of $\mathrm{Z}$ channels by using precoders for each transmitter, such that at the receiver each desired signal is orthogonal to all other signals. In Equation (8), we use

$$
\mathbf{H}_{11}^{t}(4,4)=\mathbf{H}_{1}(4,4) \mathbf{A}^{t}(4,4), \mathbf{G}_{11}^{t}(4,4)=\mathbf{G}_{1}(4,4) \mathbf{B}^{t}(4,4)
$$

to denote the equivalent channel matrices. Then Equation (8) becomes

$$
\mathbf{y}_{1}^{t}(4,1)=\mathbf{H}_{11}^{t}(4,4) \mathbf{C}(t)+\mathbf{G}_{11}^{t}(4,4) \mathbf{S}(t)+\mathbf{n}_{1}^{t}(4,1)
$$

Similarly, in Equation (10), if we use

$$
\mathbf{G}_{21}^{t}(4,4)=\mathbf{G}_{2}(4,4) \mathbf{B}^{t}(4,4)
$$

to denote the equivalent channel matrices, we have

$$
\mathbf{y}_{2}^{t}(4,1)=\mathbf{G}_{21}^{t}(4,4) \mathbf{S}(t)+\mathbf{n}_{2}^{t}(4,1)
$$

By Equation (13), since the receiver has 2 receive antennas, each symbol is actually transmitted along a 2-dimensional vector in a 2-dimensional space. Because each user sends two symbols at the same time, at the receiver, there are 4 signal vectors in the two-dimensional space.

Since we want to send $\mathbf{C}$ and $\mathbf{S}$ along orthogonal directions, we let each one of $\mathbf{C}, \mathbf{S}$ occupy only one dimension. In other words, for any codeword, we should transmit each of the corresponding four symbols in the same direction. In this way, there are only 2 transmit directions. Once we can align the 2 transmit directions of $\mathbf{C}, \mathbf{S}$ properly, we can separate them completely. This is the main idea to build the orthogonal transmission structure. Note that this is only a general idea and much details are omitted. For example, we will show later that after some array processing and moving the interference at the receiver, each symbol at each receiver will have its own direction. We need to do additional array processing to reduce the decoding complexity and achieve full diversity.

In this section, we only explain the above main idea. By Equation (13), $c_{1}, c_{2}$ are transmitted along $\mathbf{H}_{11}^{t}(4,4)(1), \mathbf{H}_{11}^{t}(4,4)(2)$, respectively. In order to make $\mathbf{H}_{11}^{t}(4,4)(1), \mathbf{H}_{11}^{t}(4,4)(2)$ along the same direction, by Equation (12), we need

$$
\mathbf{A}^{t}(4,4)(1)=\frac{1}{\alpha^{t}} \mathbf{A}^{t}(4,4)(2)
$$

where $\alpha_{11}^{t}$ is a constant that we will determine later. From $\left\|\mathbf{A}^{t}(4,4)\right\|_{F}^{2}=1$, we know

$$
\left\|\mathbf{A}^{t}(4,4)(1)\right\|_{F}^{2}=\frac{1}{1+\left(\alpha^{t}\right)^{2}}
$$


So when we design precoder $\mathbf{A}^{t}(4,4)$, Equations (16) and (17) should be satisfied. Similarly, precoders $\mathbf{B}^{t}(4,4)$ should also satisfy the following conditions:

$$
\mathbf{B}^{t}(4,4)(1)=\frac{1}{\beta^{t}} \mathbf{B}_{1}^{t}(4,4)(2)
$$

with

$$
\left\|\mathbf{B}^{t}(4,4)(1)\right\|_{F}^{2}=\frac{1}{1+\left(\beta^{t}\right)^{2}}
$$

Now Equations (13) and (15) become

$$
\begin{aligned}
& \mathbf{y}_{1}^{t}(4,1)=\left[\mathbf{H}_{11}^{t}(4,4)(1), \mathbf{H}_{11}^{t}(4,4)(1)\right] \cdot \mathbf{C}(t)+ \\
& {\left[\mathbf{G}_{11}^{t}(4,4)(1), \mathbf{G}_{11}^{t}(4,4)(1)\right] \cdot \mathbf{S}(t)+\mathbf{n}_{1}^{t}(4,1)}
\end{aligned}
$$

and

$$
\mathbf{y}_{2}^{t}(4,1)=\left[\mathbf{G}_{21}^{t}(4,4)(1), \mathbf{G}_{21}^{t}(4,4)(1)\right] \cdot \mathbf{S}(t)+\mathbf{n}_{2}^{t}(4,1)
$$

where $\mathbf{H}_{11}^{t}(4,4)(1), \mathbf{G}_{11}^{t}(4,4)(1), \mathbf{G}_{21}^{t}(4,4)(1)$ denote the first column of matrix $\mathbf{H}_{11}^{t}(4,4)$, $\mathbf{G}_{11}^{t}(4,4), \mathbf{G}_{21}^{t}(4,4)$, respectively. At receiver one, after we combine the channel equations in two time slots, we have

$$
\begin{aligned}
& \mathbf{y}_{1}(4,1)=\left(\begin{array}{cc}
\mathbf{H}_{11}^{1}(4,4)(1) & \mathbf{H}_{11}^{1}(4,4)(1) \\
\left(\mathbf{H}_{11}^{2}(4,4)(1)\right)^{*} & -\left(\mathbf{H}_{11}^{2}(4,4)(1)\right)^{*}
\end{array}\right) \cdot\left(\begin{array}{l}
c_{1} \\
c_{2}
\end{array}\right)+ \\
& \left(\begin{array}{cc}
\mathbf{G}_{11}^{1}(4,4)(1) & \mathbf{G}_{11}^{1}(4,4)(1) \\
\left(\mathbf{G}_{11}^{2}(4,4)(1)\right)^{*} & -\left(\mathbf{G}_{11}^{2}(4,4)(1)\right)^{*}
\end{array}\right) \cdot\left(\begin{array}{l}
s_{1} \\
s_{2}
\end{array}\right)+\mathbf{n}_{1}(4,1)
\end{aligned}
$$

where

$$
\mathbf{y}_{1}(4,1)=\left(\begin{array}{c}
\mathbf{y}_{1}^{1}(4,1) \\
\left(\mathbf{y}_{1}^{2}(4,1)\right)^{*}
\end{array}\right)
$$

and

$$
\mathbf{n}_{1}(4,1)=\left(\begin{array}{c}
\mathbf{n}_{1}^{1}(4,1) \\
\left(\mathbf{n}_{1}^{2}(4,1)\right)^{*}
\end{array}\right)
$$

Similarly, at receiver two, after we combine the channel equations in two time slots, we have

$$
\mathbf{y}_{2}(4,1)=\left(\begin{array}{cc}
\mathbf{G}_{21}^{1}(4,4)(1) & \mathbf{G}_{21}^{1}(4,4)(1) \\
\left(\mathbf{G}_{21}^{2}(4,4)(1)\right)^{*} & -\left(\mathbf{G}_{21}^{2}(4,4)(1)\right)^{*}
\end{array}\right) \cdot\left(\begin{array}{l}
s_{1} \\
s_{2}
\end{array}\right)+\mathbf{n}_{2}(4,1)
$$

where

$$
\mathbf{y}_{2}(4,1)=\left(\begin{array}{c}
\mathbf{y}_{2}^{1}(4,1) \\
\left(\mathbf{y}_{2}^{2}(4,1)\right)^{*}
\end{array}\right)
$$

and

$$
\mathbf{n}_{2}(4,1)=\left(\begin{array}{c}
\mathbf{n}_{2}^{1}(4,1) \\
\left(\mathbf{n}_{2}^{2}(4,1)\right)^{*}
\end{array}\right)
$$

By Equation (22), we can see that once we make vector $\mathbf{H}_{11}^{1}(4,4)(1)$ orthogonal to $\mathbf{G}_{11}^{1}(4,4)(1)$ at time slot 1 and $\mathbf{H}_{11}^{2}(4,4)(1)$ orthogonal to $\mathbf{G}_{11}^{2}(4,4)(1)$ at time slot 2 , signal vectors for $c_{1}, c_{2}$ will lie in a subspace which is orthogonal to the subspace created by the signal vectors for $s_{1}, s_{2}$. Because of this orthogonality, at the receiver one, we can easily separate the desired signals $c_{1}, c_{2}$ from the interference signals $s_{1}, s_{2}$. At Receiver 2 , since there is no interference, by Equation (25), we can easily decode the desired signals $s_{1}, s_{2}$. This is our main idea to achieve interference-free transmission in this interference channel. 
Now we show how to derive the above orthogonality by designing precoders for Users 1 and 2 simultaneously. Assume the Singular Value Decomposition of channel matrices $\mathbf{H}_{1}(4,4), \mathbf{G}_{2}(4,4)$ as follows

$$
\begin{aligned}
& \mathbf{H}_{1}(4,4)=\mathbf{V}_{H_{1}(4,4)} \Lambda_{H_{1}(4,4)} \mathbf{U}_{H_{1}(4,4)}^{\dagger} \\
& \mathbf{G}_{2}(4,4)=\mathbf{V}_{G_{2}(4,4)} \Lambda_{G_{2}(4,4)} \mathbf{U}_{G_{2}(4,4)}^{\dagger}
\end{aligned}
$$

At time slot 1, we let User 1 transmit along its best direction. In this case, we can choose the precoder

$$
\mathbf{A}^{1}(4,4)=\frac{1}{\sqrt{2}}\left[\mathbf{U}_{H_{1}(4,4)}(4,4)(1), \mathbf{U}_{H_{1}(4,4)}(4,4)(1)\right]
$$

Then we design precoders for User 2 such that at both receivers 1 , the signal vectors from User 2 are orthogonal to the signal vectors of User 1 . Note that at receiver one, the signal from User 2 is interference and at receiver two, the signal from User 2 is the desired signal. We need to consider the signals at both receivers when we design precoder for User 2. So, at time slot 1 , the precoder $\mathbf{B}^{1}$ needs to satisfy the following three equations. At receiver one, we need

$$
\left(\mathbf{H}_{11}^{1}(4,4)(1)\right)^{\dagger} \cdot \mathbf{G}_{11}^{1}(4,4)(1)=0
$$

and the power constraint

$$
\left\|\mathbf{B}^{1}(4,4)(1)\right\|^{2}=\frac{1}{2}
$$

By solving the above two equations, we can get the precoder $\mathbf{B}^{1}(4,4)$ for User 2 at time slot 1. At time slot 2, we let User 2 transmit along its best direction. In this case, we can choose the precoder

$$
\mathbf{B}^{2}(4,4)=\frac{1}{\sqrt{2}}\left[\mathbf{U}_{G_{2}(4,4)}(4,4)(1), \mathbf{U}_{G_{2}(4,4)}(4,4)(1)\right]
$$

For User 1 at time slot 2 , at receiver one, $\mathbf{A}^{2}(4,4)$ needs to satisfy the following the following equation

$$
\left(\mathbf{G}_{11}^{2}(4,4)(1)\right)^{\dagger} \cdot \mathbf{H}_{11}^{2}(4,4)(1)=0
$$

and the power constraint

$$
\left\|\mathbf{A}^{2}(4,4)(1)\right\|^{2}=\frac{1}{2}
$$

By solving the above two equations, we can get the precoder $\mathbf{A}^{2}(4,4)$ for User 1 at time slot 2. With our precoders $\mathbf{A}^{1}(4,4), \mathbf{B}^{1}(4,4)$ at time slot 1 and $\mathbf{A}^{2}(4,4), \mathbf{B}^{2}(4,4)$ at time slot 2 , we can show that we can achieve interference-free transmission with low decoding complexity and full diversity simultaneously as shown in the next two sections.

\section{Decoding with Low Complexity}

In the last section, we have shown how to build the orthogonal transmission structure. Once the orthogonal structure is built, it is easy to realize low-complexity decoding. In this section, we will show how to decode and analyze the decoding complexity. We first consider the decoding at receiver one. In Equation (22), if we let

$$
\overline{\mathbf{H}}_{1}(4,4)=\left(\begin{array}{cc}
\mathbf{H}_{11}^{1}(4,4)(1) & \mathbf{H}_{11}^{1}(4,4)(1) \\
\left(\mathbf{H}_{11}^{2}(4,4)(1)\right)^{*} & -\left(\mathbf{H}_{11}^{2}(4,4)(1)\right)^{*}
\end{array}\right)
$$


International Journal of Wireless \& Mobile Networks (IJWMN) Vol. 4, No. 2, April 2012

and

$$
\overline{\mathbf{G}}_{1}(4,4)=\left(\begin{array}{cc}
\mathbf{G}_{11}^{1}(4,4)(1) & \mathbf{G}_{11}^{1}(4,4)(1) \\
\left(\mathbf{G}_{11}^{2}(4,4)(1)\right)^{*} & -\left(\mathbf{G}_{11}^{2}(4,4)(1)\right)^{*}
\end{array}\right)
$$

then Equation (22) becomes

$$
\mathbf{y}_{1}(4,1)=\overline{\mathbf{H}}_{1}(4,4) \cdot\left(\begin{array}{l}
c_{1} \\
c_{2}
\end{array}\right)+\overline{\mathbf{G}}_{1}(4,4) \cdot\left(\begin{array}{l}
s_{1} \\
s_{2}
\end{array}\right)+\mathbf{n}_{1}(4,1)
$$

Note that at receiver one, $\left(\begin{array}{l}c_{1} \\ c_{2}\end{array}\right)$ are the desired signal and $\left(\begin{array}{l}s_{1} \\ s_{2}\end{array}\right)$ are the interference. We can cancel the interference by multiplying both sides of Equation (38) by matrix $\overline{\mathbf{H}}_{1}(4,4)^{\dagger}$. Then we get

$$
\overline{\mathbf{H}}_{1}(4,4)^{\dagger} \cdot \mathbf{y}_{1}(4,1)=\overline{\mathbf{H}}_{1}(4,4)^{\dagger} \overline{\mathbf{H}}_{1}(4,4)\left(\begin{array}{l}
c_{1} \\
c_{2}
\end{array}\right)+\overline{\mathbf{H}}_{1}(4,4)^{\dagger} \mathbf{n}_{1}(4,1)
$$

Here we have canceled the interference because $\overline{\mathbf{H}}_{1}(4,4)^{i} \overline{\mathbf{G}}_{1}(4,4)=0$. In order to decode the symbols, we first multiply both sides of Equations (39) by matrix $\left(\overline{\mathbf{H}}_{1}(4,4)^{\dagger} \overline{\mathbf{H}}_{1}(4,4)\right)^{-\frac{1}{2}}$ to whiten the noise, i.e.,

$$
\begin{aligned}
& \left(\overline{\mathbf{H}}_{1}(4,4)^{\dagger} \overline{\mathbf{H}}_{1}(4,4)\right)^{-\frac{1}{2}} \overline{\mathbf{H}}_{1}(4,4)^{\dagger} \cdot \mathbf{y}_{1}(4,1)= \\
& \left(\overline{\mathbf{H}}_{1}(4,4)^{\dagger} \overline{\mathbf{H}}_{1}(4,4)\right)^{\frac{1}{2}}\left(\begin{array}{c}
c_{1} \\
c_{2}
\end{array}\right)+ \\
& \left(\overline{\mathbf{H}}_{1}(4,4)^{\dagger} \overline{\mathbf{H}}_{1}(4,4)\right)^{-\frac{1}{2}} \overline{\mathbf{H}}_{1}(4,4)^{\dagger} \mathbf{n}_{1}(4,1)
\end{aligned}
$$

Then we can detect $\left(c_{1}, c_{2}\right)$ by

$$
\begin{aligned}
& \widehat{c}_{1}, \widehat{c}_{2}= \\
& \arg \min _{c_{1}, c_{2}} \|\left(\overline{\mathbf{H}}_{1}(4,4)^{\dagger} \overline{\mathbf{H}}_{1}(4,4)\right)^{-\frac{1}{2}} \overline{\mathbf{H}}_{1}(4,4)^{\dagger} \\
& \times \mathbf{y}_{1}(4,1)-\left(\overline{\mathbf{H}}_{1}(4,4)^{\dagger} \overline{\mathbf{H}}_{1}(4,4)\right)^{\frac{1}{2}}\left(\begin{array}{c}
c_{1} \\
c_{2}
\end{array}\right) \|_{F}^{2}
\end{aligned}
$$

Further, note that

$$
\begin{aligned}
& \overline{\mathbf{H}}_{1}(4,4)^{\dagger} \overline{\mathbf{H}}_{1}(4,4)= \\
& \left(\begin{array}{ll}
\left\|\mathbf{H}_{11}^{1}(4,4)(1)\right\|\left\|^{2}+\right\| \mathbf{H}_{11}^{2}(4,4)(1) \|^{2} & \left\|\mathbf{H}_{11}^{1}(4,4)(1)\right\|\left\|^{2}-\right\| \mathbf{H}_{11}^{2}(4,4)(1) \|^{2} \\
\left\|\mathbf{H}_{11}^{1}(4,4)(1)\right\|^{2}-\left\|\mathbf{H}_{11}^{2}(4,4)(1)\right\|^{2} & \left\|\mathbf{H}_{11}^{1}(4,4)(1)\right\|^{2}+\left\|\mathbf{H}_{11}^{2}(4,4)(1)\right\|^{2}
\end{array}\right)
\end{aligned}
$$

So, when QAM is used, Equation (40) is equivalent to the following two equations.

$$
\begin{aligned}
& \operatorname{Real}\left\{\left(\overline{\mathbf{H}}_{1}(4,4)^{\dagger} \overline{\mathbf{H}}_{1}(4,4)\right)^{-\frac{1}{2}} \overline{\mathbf{H}}_{1}(4,4)^{\dagger} \cdot \mathbf{y}_{1}(4,1)\right\} \\
& =\left(\overline{\mathbf{H}}_{1}(4,4)^{\dagger} \overline{\mathbf{H}}_{1}(4,4)\right)^{\frac{1}{2}} \operatorname{Real}\left\{\left(\begin{array}{c}
c_{1} \\
c_{2}
\end{array}\right)\right\} \\
& +\operatorname{Real}\left\{\left(\overline{\mathbf{H}}_{1}(4,4)^{\dagger} \overline{\mathbf{H}}_{1}(4,4)\right)^{-\frac{1}{2}} \overline{\mathbf{H}}_{1}(4,4)^{\dagger} \mathbf{n}_{1}(4,1)\right\} \\
& \operatorname{Imag}\left\{\left(\overline{\mathbf{H}}_{1}(4,4)^{\dagger} \overline{\mathbf{H}}_{1}(4,4)\right)^{-\frac{1}{2}} \overline{\mathbf{H}}_{1}(4,4)^{\dagger} \cdot \mathbf{y}_{1}(4,1)\right\} \\
& =\left(\overline{\mathbf{H}}_{1}(4,4)^{\dagger} \overline{\mathbf{H}}_{1}(4,4)\right)^{\frac{1}{2}} \operatorname{Imag}\left\{\left(\begin{array}{c}
c_{1} \\
c_{2}
\end{array}\right)\right\} \\
& +\operatorname{Imag}\left\{\left(\overline{\mathbf{H}}_{1}(4,4)^{\dagger} \overline{\mathbf{H}}_{1}(4,4)\right)^{-\frac{1}{2}} \overline{\mathbf{H}}_{1}(4,4)^{\dagger} \mathbf{n}_{1}(4,1)\right\}
\end{aligned}
$$


International Journal of Wireless \& Mobile Networks (IJWMN) Vol. 4, No. 2, April 2012

So we can detect the real part and the imaginary part of $c_{1}, c_{2}$ separately as follows:

$$
\begin{aligned}
& \operatorname{Real}\left\{\widehat{c}_{1}, \widehat{c}_{2}\right\}=\arg \min _{\operatorname{Real}\left\{c_{1}, c_{2}\right\}} \\
& \| \operatorname{Real}\left\{\left(\overline{\mathbf{H}}_{1}(4,4)^{\dagger} \overline{\mathbf{H}}_{1}(4,4)\right)^{-\frac{1}{2}} \overline{\mathbf{H}}_{1}(4,4)^{\dagger} \cdot \mathbf{y}_{1}(4,1)\right\} \\
& -\left(\overline{\mathbf{H}}_{1}(4,4)^{\dagger} \overline{\mathbf{H}}_{1}(4,4)\right)^{\frac{1}{2}} \operatorname{Real}\left\{\left(\begin{array}{c}
c_{1} \\
c_{2}
\end{array}\right)\right\} \|_{F}^{2} \\
& \operatorname{Imag}\left\{\widehat{c}_{1}, \widehat{c}_{2}\right\}=\arg \min _{\operatorname{Imag}\left\{c_{1}, c_{2}\right\}} \\
& \| \operatorname{Imag}\left\{\left(\overline{\mathbf{H}}_{1}(4,4)^{\dagger} \overline{\mathbf{H}}_{1}(4,4)\right)^{-\frac{1}{2}} \overline{\mathbf{H}}_{1}(4,4)^{\dagger} \cdot \mathbf{y}_{1}(4,1)\right\} \\
& -\left(\overline{\mathbf{H}}_{1}(4,4)^{\dagger} \overline{\mathbf{H}}_{1}(4,4)\right)^{\frac{1}{2}} \operatorname{Imag}\left\{\left(\begin{array}{c}
c_{1} \\
c_{2}
\end{array}\right)\right\} \|_{F}^{2}
\end{aligned}
$$

The decoding complexity is symbol-by-symbol. Similarly, we can detect $s_{1}, s_{2}$ with symbol-bysymbol complexity at receiver two.

\section{Diversity Analysis}

In this section, we show that our proposed scheme can achieve full diversity for each user. We only prove that at receiver 1 , the diversity for $c 1, c 2$ from user 1 is full. The proof for $s_{1}, s_{2}$ at receiver two will be similar. First, the diversity is defined as

$$
d=-\lim _{\rho \rightarrow \infty} \frac{\log P_{e}}{\log \rho}
$$

where $\rho$ denotes the SNR and $P_{e}$ represents the probability of error. We let $\mathbf{e}=\left(\begin{array}{l}e_{1} \\ e_{2}\end{array}\right)=$ $\left(\begin{array}{l}c_{1} \\ c_{2}\end{array}\right)-\left(\begin{array}{l}\widehat{c}_{1} \\ \widehat{c}_{2}\end{array}\right)$ denote the error vector. Here we add a rotation matrix $\mathbf{R}$ on the transmitted codewords to improve the system performance. Based on Equation (40), the pairwise error probability (PEP) for $c_{1}, c_{2}$ can be written as [18]

$$
\begin{aligned}
& P\left(\mathbf{c} \rightarrow \overline{\mathbf{c}} \mid \overline{\mathbf{H}}_{1}(4,4)\right)= \\
& Q\left(\sqrt{\frac{\rho\left\|\left(\overline{\mathbf{H}}_{1}(4,4)^{\dagger} \overline{\mathbf{H}}_{1}(4,4)\right)^{\frac{1}{2}} \mathbf{R}(4,4) \mathbf{e}(4,1)\right\|_{F}^{2}}{4}}\right)= \\
& Q\left(\sqrt{\frac{\mathbf{e}(4,1)^{\dagger} \mathbf{R}(4,4)^{\dagger} \overline{\mathbf{H}}_{1}(4,4)^{\dagger} \overline{\mathbf{H}}_{1}(4,4) \mathbf{R}(4,4) \mathbf{e}(4,1)}{4 / \rho}}\right) \\
& \leq \exp ( \\
& \left.\frac{\mathbf{e}(4,1)^{\dagger} \mathbf{R}(4,4)^{\dagger} \overline{\mathbf{H}}_{1}(4,4)^{\dagger} \overline{\mathbf{H}}_{1}(4,4) \mathbf{R}(4,4) \mathbf{e}(4,1)}{-4}\right) \\
& =\exp \left(-\frac{\rho \lambda}{4 / \rho}\right)
\end{aligned}
$$

where

$$
\lambda=|| \mathbf{H}_{11}^{1}(4,4)(1)||_{F}^{2}\left|\widehat{e}_{1}+\widehat{e}_{2}\right|^{2}+|| \mathbf{H}_{11}^{2}(4,4)(1)||_{F}^{2}\left|\widehat{e}_{1}-\widehat{e}_{2}\right|^{2}
$$

and 


$$
\widehat{\mathbf{e}}(4,1)=\left(\begin{array}{c}
\widehat{e}_{1} \\
\widehat{e}_{2}
\end{array}\right)=\mathbf{R}(4,4) \mathbf{e}(4,1)
$$

Since

$$
\left\|\mathbf{H}_{11}^{1}(4,4)(1)\right\|_{F}^{2} \geq \frac{\left\|\mathbf{H}_{1}(4,4)\right\|_{F}^{2}}{2} \cdot \frac{1}{2}
$$

Inequality (48) can be written as

$$
\begin{aligned}
& P\left(\mathbf{c} \rightarrow \overline{\mathbf{c}} \mid \overline{\mathbf{H}}_{1}(4,4)\right) \leq \exp \left(-\frac{\rho \lambda}{4}\right) \\
& =\exp \left(-\frac{\rho|| \mathbf{H}_{1}(4,4)||_{F}^{2}\left|\widehat{e}_{1}+\widehat{e}_{2}\right|^{2}}{16}\right)
\end{aligned}
$$

Therefore, we have

$$
\begin{aligned}
& P(\mathbf{c} \rightarrow \overline{\mathbf{c}})=E\left[P\left(\mathbf{c} \rightarrow \overline{\mathbf{c}} \mid \overline{\mathbf{H}}_{1}(4,4)\right)\right] \\
& =E\left[\exp \left(-\frac{\rho|| \mathbf{H}_{1}(4,4) \|_{F}^{2}\left|\widehat{e}_{1}+\widehat{e}_{2}\right|^{2}}{16}\right)\right] \\
& =\frac{1}{\prod_{j=1}^{4}\left(1+\frac{\rho \tau}{16}\right)}
\end{aligned}
$$

Where

$$
\tau=\left|\widehat{e}_{1}+\widehat{e}_{2}\right|^{2}
$$

At high SNR region, (53) can be written as

$$
P(\mathbf{c} \rightarrow \overline{\mathbf{c}}) \leq\left(\frac{\rho \tau}{16}\right)^{-4}
$$

So the diversity is 4 , full diversity, as long as $\tau \neq 0$. Also the coding gain is affected by $\tau$ and we can choose rotation matrix $\mathbf{R}(4,4)$ properly to maximize $\tau$. The best choice for rotation matrix depends on the adopted constellation. Such an optimization is a straightforward optimization that has been discussed in many existing literature [19]. Similarly, we can prove that the diversity for other codewords is also full.

\section{Simulation Results}

In this section, we provide simulation results to evaluate the performance of the proposed scheme. First, we assume there are 2 transmitters each with 4 transmit antennas and 2 receivers each with 4 antennas. Each user uses our proposed scheme to transmit Alamouti codes to its receiver. Figure 2 presents simulation results using QPSK. We compare the performance of our scheme with that of two other scenarios that can achieve interference cancellation. In the first scenario, we use TDMA and beamforming. That is, at each time slot, only one transmitter sends signals to one receiver using beamforming. 16-QAM is used to have the same bit-rate. In the second scenario, each user uses the multi-user detection (MUD) method to send its codewords. The results show that our proposed scheme can achieve full diversity and symbol rate one. Note that we combine the array processing and space-time coding to avoid symbol rate loss. This does not mean that we cannot change the bit rate. We can always adapt the bit rate by changing the constellation according to the channel condition. In comparison, the TDMA and beamforming method can achieve full diversity but the rate is one half. The MUD method can achieve full rate, but it cannot achieve full diversity. As shown in the figure, our scheme 
provides the best performance due to its high diversity and increased coding gain without any rate loss.

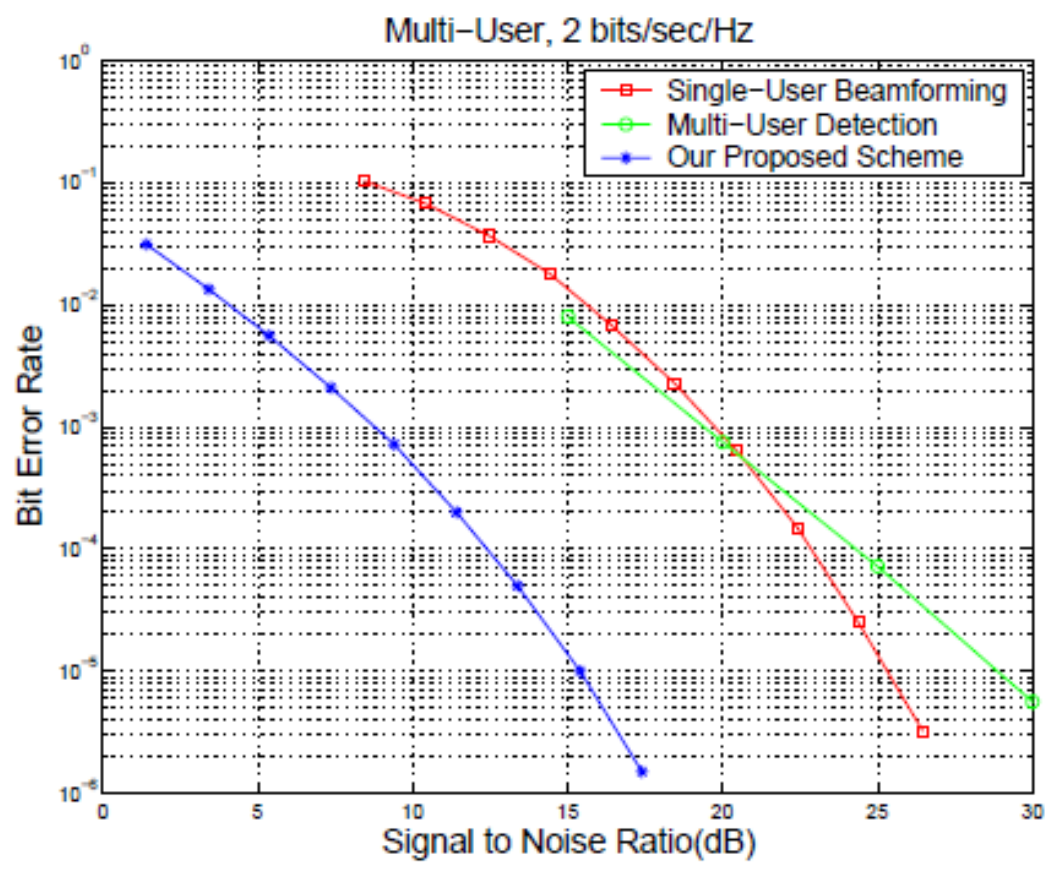

Figure 2: Simulation results for 2 users each with 4 transmit antennas and 2 receivers each with 4 receive antennas. The constellation is QPSK.

\section{Conclusions}

In this paper, we propose an efficient transmission scheme for MIMO multi-user channels with two transmitters each with four transmit antennas and two receivers each with four receive antennas. By combining array processing and space-time coding, we can achieve full diversity and low decoding complexity. We analytically prove that our scheme can achieve lowcomplexity decoding and full diversity. Simulation results validate our theoretical analysis.

\section{REFERENCES}

[1] A. Zaier and R. Bouallegue, Blind Channel Estimation Enhancement for MIMO- OFDM Systems Under High Mobility Conditions, International Journal of Wireless \& Mobile Networks (IJWMN), 2012.

[2] F. Li, "Optimization of input covariance matrix for multi-antenna correlated channels," International Journal of Computer Networks \& Communications (IJCNC), 2011.

[3] D. C. Popescu, O. Popescu, and C. Rose, "Interference avoidance for multi access vector channels," in Proc. Int. Symp. Inform. Theory, July 2002, p. 499.

[4] J. Wang and K. Yao, "Multiuser spatio-temporal coding for wireless communications," in Proc. IEEE Wireless Commun. Networking Conf., vol. 1, Mar. 2002, pp. 276-279.

[5] G. G. Raleigh and J.M. Cioffi, "Spatio-temporal coding for wireless communication," IEEE Trans. Commun., vol. 46, pp. 357-366, Mar. 1998.

[6] H. Lu, R. Vehkalahti, C. Hollanti, J. Lahtonen, Y. Hong and E. Viterbo, "New space time code constructions for two-user multiple access channels," IEEE J. Sel. Top. Sign. Proces., vol. 3, no. 6, pp.939-957, Dec. 2009. 
International Journal of Wireless \& Mobile Networks (IJWMN) Vol. 4, No. 2, April 2012

[7] F. Li and H. Jafarkhani, "Space-Time Processing for X Channels Using Precoders," IEEE Transactions on Signal Processing.

[8] F. Li, Multi-Antenna Multi-User Interference Cancellation and Detection Using Precoders, PhD thesis, UC Irvine, 2012

[9] P. Wolniansky, G. Foschini, G. Golden, and R. Valenzuela, "V-BLAST: An architecture for realizing very high data rates over the rich scattering wireless channel," in Proc. ISSSE, Sept. 1998.

[10] G. J. Foschini and M. J. Gans, "On limits of wireless personal communications in a fading environment when using multiple antennas," Wireless Pers. Commun., vol. 6, pp. 311-335, Mar. 1998.

[11] A. F. Naguib, N. Seshadri, and A. R. Calderbank, "Applications of space-time block codes and interference suppression for high capacity and high data rate wireless systems," in Proc. Asilomar Conf. Signals, Systems and Computers, 1998.

[12] F. Li and H. Jafarkhani, "Multiple-antenna interference cancellation and detection for two users using quantized feedback," IEEE Transactions on Wireless Communication, vol. 10, no. 1, pp. 154163, Jan 2011.

[13] F. Li and H. Jafarkhani, "Interference cancellation and detection for multiple access channels with four users," in Proceedings of IEEE International Conference on Communications(ICC 2010), June 2010.

[14] A. Stamoulis, N. Al-Dhahir and A. R. Calderbank, "Further results on interference cancellation and space-time block codes," in Proc. 35th Asilomar conf. on Signals, Systems and Computers, pp. 257262, Oct. 2001.

[15] F. Li and H. Jafarkhani, "Interference Cancellation and Detection Using Precoders," IEEE International Conference on Communications (ICC 2009), June 2009.

[16] S.Karmakar, M. K. Varanasi, "The diversity-multiplexing tradeoff of the MIMO Z interference channel,” Proc. IEEE int. Symp. on Inform. Theory, pp 2188-2192, Jul. 2008.

[17] C. Sun, N.C. Karmakar, K.S. Lim, A. Feng, "Combining beamforming with Alamouti scheme for multiuser MIMO communications," in Proceedings of Vehicular Technology Conference, 2004.

[18] J. Huang, E. Au, and V. Lau, "Precoding of space-time block codes in multiuser MIMO channels with outdated channel state information," in Proceedings of the IEEE International Symposium on Information Theory (ISIT '07), June 2007.

[19] F. Li, “Array Processing for Multi-User Multi-Antenna Interference Channels Using Precoders," Wireless Personal Communications, 2012.

[20] F. Li and H. Jafarkhani, "Using quantized feedback to cancel interference in multiple access channels," in Proceedings of IEEE Global Telecommunications Conference(Globecom 2010), December, 2010.

[21] E. Malkam and H. Leib, "Coded diversity on block-fading channels," IEEE Trans. Inf. Th., vol. 45, no. 2, Mar. 1999.

[22] L. Ozarow, S. Shamai, and A. D. Wyner, "Information theoretic considerations for cellular mobile radio,” IEEE Trans. Veh. Technol., vol. 43, pp. 359-378, May 1994.

[23] V. R. Cadambe and S. A. Jafar, "Interference alignment and the degrees of freedom for the K user interference channel," IEEE Transactions on Information Theory, vol. 54, no. 8, pp. 3425-3441, Aug 2008.

[24] F. Li and Q. T. Zhang, "Transmission strategy for MIMO correlated rayleigh fading channels with mutual coupling," in Proceedings of IEEE International Conference on Communications (ICC 2007), June, 2007.

[25] F. Li and H. Jafarkhani, "Resource allocation algorithms with reduced complexity in MIMO multihop fading channels," in Proceedings of IEEE Wireless Communications and Networking Conference, 2009. 
International Journal of Wireless \& Mobile Networks (IJWMN) Vol. 4, No. 2, April 2012

[26] S. M. Alamouti, “A simple transmit diversity technique for wireless communications," IEEE J. Select. Areas Commun., vol. 16, no. 8, pp. 1451-1458, Oct. 1998.

[27] M. K. Simon and M.-S. Alouini, Digital Communication over Fading Channels, $1^{\text {st }}$ ed. New York: Wiley, 2000.

[28] F. Li and H. Jafarkhani, "Multiple-antenna interference cancellation and detection for two users using precoders," IEEE Journal of Selected Topics in Signal Processing, December 2009.

[29] F. Li and H. Jafarkhani, "Interference Cancellation and Detection for More than Two Users," IEEE Transactions on Communications, March 2011.

[30] E. Bayer-Fluckiger, F. Oggier, and E. Viterbo, "New algebraic constructions of rotated Zn-lattice constellations for the Rayleigh fading channel," IEEE Trans. Inform. Theory, vol. 50, pp. 702-714, Apr. 2004. 